\title{
Generality of the Simple Averaging model in personality impression formation'
}

JOHN T. PARTINGTON

UNIVERSITY OF WESTERN ONTARIO, LONDON

Ss made numerical impression ratings for hypothetical stimulus people represented by verbal information. Effects of a wide array of stimulus parameters were evaluated including vehicle of information, denotative content, and such quantitative characteristics as amount, polarity, and complexity. The Simple Averaging model provided a good approximation to these data.

The present investigation seeks to evaluate an arithmetic model of personality impression formation under relatively wider stimulus conditions than previously employed.

To date there is ample empirical evidence supporting Arithmetic models of personality impression formation, for example, Simple Averaging (Anderson, 1962), Adding (Fishbein \& Hunter, 1964), and Weighted Averaging (Anderson, 1965). The lawfulness demonstrated by these studies is impressive when contrasted with Asch's (1946) gestalt formulation of impression formation. However, greater confidence might be placed in contemporary arithmetic models if more representative designs had been employed in studies which support them. Consider first that in many recent studies stimulus people were described by sets of trait adjectives. Conclusions from such studies would seem to be limited since trait adjectives are distillations of other more elementary forms of stimulus information such as observable behavior. A second possible shortcoming of certain previous studies is that "goodness of fit" between stimulus input and impression response was assessed along purely evaluative dimensions, e.g., likeability, rather than along a more representative array of denotative dimensions.

\section{Method}

This investigation involved two experimental treatments, a Trait Set and a Statement Set of hypothetical stimulus people represented by verbal information. Each treatment required Ss to form impressions of these hypothetical people. Forty male undergraduate psychology students were assigned randomly to each treatment. The group assigned to the Trait Set were asked to rate a set of 20 hypothetical people described by trait adjectives (e.g., leading, powerful). The group assigned to the Statement Set rated a parallel set represented by self-referent statements (e.g., I like to direct the activities of others. I usually win in arguments.). Both traits and statements were derived from item pools developed by Jackson (1966) for construction of the Personality Research Form.

Each set of stimulus people included four sub- groups and each sub-group comprised five hypothetical people described along a particular personality dimension. The four denotative dimensions were Dominance, Affiliation, Order, and Endurance. Within each denotative sub-group stimulus people differed in terms of the amount, polarity, and complexity of information used to represent them. Specifically, the design for quantitatively varying stimulus people in each denotative sub-group was as follows: One "high $\left(\mathrm{H}^{+}\right)^{\text {" }}$ stimulus person described by six items of univalent information characteristic of one pole of a personality dimension, another "high $\left(\mathrm{H}^{-}\right)$" person described by six univalent items characteristic of the opposite pole of the same dimension, one "medium $\left(\mathrm{M}^{+}\right)$" person described by three univalent items from one of these poles, and two "mixed $\left(\mathrm{Mx}^{+-}\right.$and $\left.\mathrm{Mx}^{-+}\right)$" stimulus people described with bivalent information. For each of these more complex people, three information items represented one pole of the dimension, and three the other. These two people differed in terms of the order in which the information was presented in their descriptions. Rules followed in the construction of the stimulus people and individual item weights are reported elsewhere (Partington, 1966).

Testing was conducted in small groups. Before each session, an attempt was made to create a realistic set by playing a tape recorded interview and then showing slides containing traits or statements describing the interviewee. The experimental stimulus slides were then shown. Ss were required to rate each stimulus person on six bipolar personality dimensions. In each case only one response scale was directly relevant to the denotative meaning of the information presented.

Results

The primary data are impression ratings of hypothetical people on denotative response scales relevant to the stimulus information representing each hypothetical stimulus person. These ratings are to be contrasted with those predicted from an arithmetic model of impression formation. In quantitative terms, the hypothesis is that the obtained impression ratings (i.e., average group ratings) are a linear function of those predicted from the Simple Averaging model (i.e., average ratings of the component units of information representing each stimulus person). This was tested by correlating the obtained and predicted impression ratings across the 20 stimulus people in each stimulus set. These correlations were .993 and .976 for the Trait and Statement Sets, respectively. This demonstrates that 

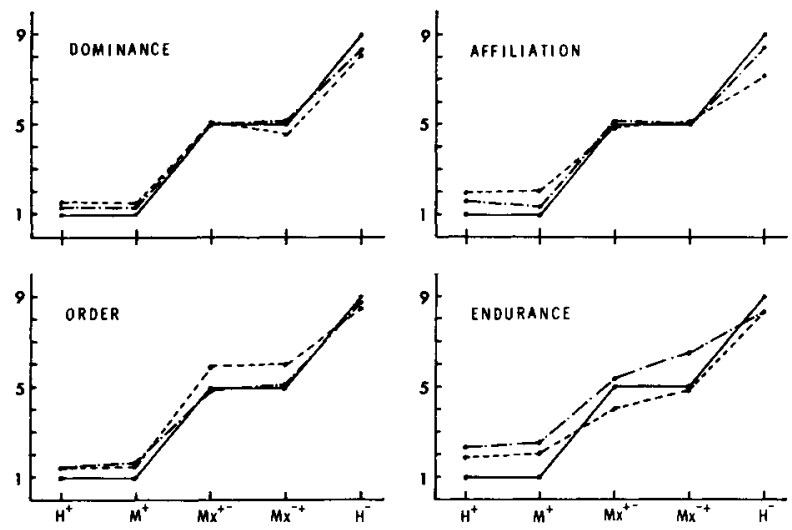

Fig. 1. Predicted and obtained impression values of stimulus people.

the Simple Averaging model accounts for these data very well.

Specific effects of quantitative parameters of stimulus information were examined by four separate analyses of variance, one for each denotatively defined sub-group of stimulus people.

Main effects due to quantitative differences across the five levels of stimulus people were statistically significant in each of the four separate analyses ( $F=$ Dominance:323.5; Affiliation: 282.3; Order: 366.3; and Endurance: $213.3, \mathrm{df}=4 / 312, \mathrm{p}<.01$ ). Figure 1 shows predicted scale values (solid lines) and obtained mean ratings for stimulus people in the Statement and Trait Sets (broken and dotted lines, respectively). As may be seen, findings are consistent with expectations from the Simple Averaging model. For instance, Newman-Keuls comparisons between means for the high and medium stimulus people on each of the four personality dimensions, for both the Statement and Trait conditions, show no significant rating differences. Furthermore, mean ratings for the pair of mixed stimulus people on each dimension do not differ significantly, and, as expected, vary around the neutral rating of "five."

Main effects and interactions associated with vehicle of stimulus information (i.e., traits vs statements) showed that there was little difference between stimulus forms. The only significant Trait Set vs Statement Set difference occurred in the analysis of ratings for stimulus people described on the Endurance dimension $(F=18.54, \mathrm{df}=1 / 78, \mathrm{p}<.01)$.

\section{Discussion}

The most obvious empirical generalization from the above results is that the Simple Averaging model provides a good approximation to peoples' impression formation strategies. Furthermore, this seems to be true for impressions of stimulus people represented by information varied across a relatively broad array of denotative and quantitative stimulus parameters. However, a note of caution should be raised. This study, like many previous ones, infers support for an Arithmetical model from ratings along predetermined, unidimensional, numerical scales. Such an inference rests on several assumptions which warrant careful scrutiny. Consider the following questions. Can the $\mathrm{E}$ actually choose the dimension relevant to a given social stimulus domain? Do Ss understand what is implied by the given response dimension? Do Ss actually use the rating scale in an equal appearing intervals manner? Can numerical ratings be considered representative of the complexity of Ss' individual impression strategies? It is felt that these and many other questions must be answered by future work before firm conclusions can be made about the explanatory value of arithmetic models of personality impression formation. Regardless of these qualifications, however, the consistency of findings in the present study suggests that the Simple Averaging model provides a good approximation to numerical impression ratings.

\section{References}

Anderson, N. H. Application of an additive model to impression formation. Science, 1962 (No. 3542), 817-818.

Anderson, N. H. Averaging versus adding as a stimulus combination rule in impression formation. J. exp. Psychol., 1965, 70, 394-400.

Asch, S. E. Forming impressions of personality. J, abnorm. soc. Psychol.; 1946, 41, 258-290.

Fishbein, M., \& Hunter, Ronda. Summation versus balance in attitude organization and change. J. abnorm. soc. Psychol., 1964, $69,505-510$.

Jackson, D. N. A modern strategy for personality assessment: The Personality Research Form. London: The University of Western Ontario, Research Bulletin No. 30, 1966.

Partington, J, T. A test of averaging, adding, and dynamic models in personality impression formation. Unpublished Doctoral Dissertation. London: The University of Western Ontario, 1966.

\section{Note}

1. I wish to thank Douglas N. Jackson for his continued guidance throughout this study. 